\title{
Hydrogen Generation Potential Through Excess and Curtailed Energy from the Major Ontario Grid System
}

\author{
Mudit Nijhawan ${ }^{1 *}$, Sandra E. Guido ${ }^{1}$, Natalina Mussio ${ }^{1}$, Peter J. Kriemadis ${ }^{1}$, Ofelia A. Jianu ${ }^{1}$ \\ ${ }^{1}$ Meachnical, Automotive, and Materials Engineering, University of Windsor, Windsor, Canada \\ *nijhawam@uwindsor.ca
}

\begin{abstract}
Global warming is amongst the biggest threats being faced by humanity, and climate change resulting from rising temperatures have created extreme weather events resulting in a loss of life and adverse economic events. To reduce the likelihood of such events, political institutions have agreed to reduce the warming rate through the reduction of carbon emissions by adopting renewable energy producing technologies. The adoption of renewable energy and nuclear power has allowed Ontario to produce electricity at amongst the lowest rates of carbon emissions. The majority of Ontario's electricity is produced by nuclear (60\%), hydro $(26 \%)$, wind $(7 \%)$, natural gas $(5 \%)$, solar $(1 \%)$ and the remaining being produced by biofuel, geothermal and petroleum electricity generation sources. Supply and demand for electricity is rarely equal and renewables such as wind and solar can be very intermittent as they are dependent on weather patterns, as such grid operators vary the supply of electricity to the grid by stalling wind turbines or disconnecting solar panels. An issue also arises when the demand increases above the supply threshold of renewables, at which point fossil fuel-based electricity generators are employed to supply the peak demand. This is in part due to the inflexibility of the grid due to a lack of a major electricity storage medium. The following research explores the potentials of using hydrogen as a storage of excess grid electricity, with the presumption that hydrogen will be used to supplement the gird during times of high demand in the same means as natural gas and other fossil fuel electricity sources. The research also explored the potential hydrogen that can be generated from solar and wind energy generators that have been curtailed due to a lack of electricity demands. The research considered the generation of hydrogen using a PEM electrolyzer and also explores the longterm impact of hydrogen as an energy source. This is performed through a life cycle analysis, which investigates the initial input of raw feedstock materials, along with the impact of maintenance and finally the disposal of such a system. Results obtained include the production of hydrogen along with estimated curtailed and excess electricity and the emissions of greenhouse gases over the lifetime of such a system.
\end{abstract}

